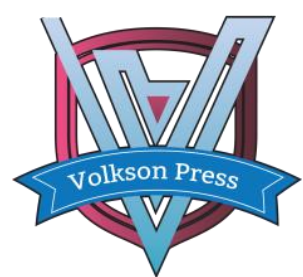

Contents List available at VOLKSON PRESS

Economics \& Management Innovations(EMI)

DOI : http://doi.org/10.26480/icemi.01.2017.01.05

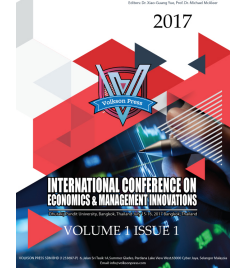

\title{
The Greeks of the Piterbarg option pricing framework
}

\section{Coenraad C. A. Labuschagne ${ }^{1,2}$, Sven T. von Boetticher ${ }^{3}$}

${ }^{1}$ Department of Finance and Investment Management, University of Johannesburg, PO Box 524, Aucklandpark 2006, South Africa.

${ }^{2}$ The contribution of the first named author is based on research supported by the National Research

Foundation, Grant Number 87502.

${ }^{3}$ Department of Finance and Investment Management, University of Johannesburg, PO Box 524, Aucklandpark 2006, South Africa.

${ }^{1}$ Email: Coenraad.Labuschagne@gmail.com.

3Email: vonboettichert@gmail.com

This is an open access article distributed under the Creative Commons Attribution License, which permits unrestricted use, distribution, and reproduction in any medium, provided the original work is properly cited.

\section{ARTICLE DETAILS}

\section{Article History:}

Received 02 october 2017 Accepted 06 october 2017 Available online 11 october 2017

\section{Keywords:}

Piterbarg model, Greeks, option pricing, delta, gamma, vega, rho, theta

\section{ABSTRACT}

In this paper the Greeks are derived in the Piterbarg option pricing framework, which derives the price of an option through three unique interest rates, and collateral payments. The different scenarios of collateral payments are discussed, and closed form solutions for the option prices are derived. The Greeks are found for each scenario and implemented.

\section{Introduction}

The Global Financial Crisis (GFC) emphasised the need for improved management of risk by financial institutions that sell options in overthe-counter markets. If a financial institution sells an option, there is risk involved and the financial institution usually hedges the exposure to reduce the risk. One of the methods available to hedge the position is by meansof the Greeks.

The Greeks give the sensitivity of the price of an options to a change in underlying parameters on which the value of the option depends. They are also called the risk sensitivities, risk measures or hedge parameters. Therefore Greeks provide information with regards to hedging an option. The different Greeks that are used, measure different dimensions in the risk to an option position. In practice, appropriate management of the different Greeks provide acceptable risk levels.

Traditionally financial institutions use the Black-Scholes-Merton (BSM) model for option pricing. The Greeks are described in terms of partial derivatives of the option price function with respect to the parameters that the option price function depends on. For example, Delta which is the first partial derivative with respect to the underlying asset, indicates the change in the option's value given a change in the price of the underlying asset. This measure is used when a trading desk seeks to hedge out their market risk.

The BSM model relies on assumptions that the GFC exposed as oversimplified. One of these assumptions is the use of one interest rate, namely the risk-free rate.

Post the GFC, Piterbarg introduced an option pricing model which uses a regime of interest rates, and which reduces to the BSM model in a special case. In the Piterbarg framework, risk management by means of the Greeks, requires the extension of the Greeks to the Piterbarg model and an analysis of the Greeks. This is the objective of this paper.

\section{BSM model}

The Black-Scholes-Merton (BSM) option pricing model has been widely used to value European call and put options on non-dividend paying stocks. The option valuation depends on the price of the underlying $\mathrm{S}$, the risk-free interest rate $r$, the volatility of the underlying $\sigma$, maturity of the contract $\mathrm{T}$, and the strike price K. Under various assumption, which include that the stock price $\mathrm{S}$ follows geometric Brownian motion with constant volatility $\sigma$, and the risk-free rate $r$ is constant, the BSM model is described by the following partial differential equation (PDE):

$$
\frac{\partial V}{\partial t}+r S \frac{\partial V}{\partial S}+\frac{1}{2} \sigma^{2} S^{2} \frac{\partial^{2} V}{\partial S^{2}}=r V
$$

where $\mathrm{V}$ is the price of a derivative which is contingent on $\mathrm{S}$ and time $\mathrm{t} \in$ $[0, \mathrm{~T}]$.

For a European call and put option with strike K, the BSM PDE has an explicit Solution

$$
V=\alpha\left(S_{0} \mathcal{N}\left(\alpha d_{1}\right)-K e^{-r(T-t)} \mathcal{N}\left(\alpha d_{2}\right)\right),
$$

Where

$$
d_{1}=\frac{\ln \left(\frac{S_{0}}{K}\right)+\left(r+\frac{1}{2} \sigma^{2}\right)(T-t)}{\sigma \sqrt{(T-t)}} \text { and } d_{2}=d_{1}-\sigma \sqrt{T-t},
$$

$\alpha=1$ for a call option and $\alpha=-1$ for a put option, and $\mathrm{N}(\mathrm{x})$ is the cumulative distribution function of the standard normal distribution. The Greeks, Delta, Gamma, Vega, Theta and Rho are respectively defined by

$$
\Delta=\frac{\partial V}{\partial S}, \quad \Gamma=\frac{\partial^{2} V}{\partial^{2} S}, \quad \nu=\frac{\partial V}{\partial \sigma}, \quad \Theta=\frac{\partial V}{\partial t} \text { and } \rho=\frac{\partial V}{\partial r} .
$$


The Greeks are described in terms of partial derivatives of the option price $V$ with respect to the parameters $S, \sigma, t$, and $r$ that $V$ depends on. As the BSM PDE has a closed form solution, it is possible to calculate the Greeks from their explicitly descriptions.

The Global Financial Crisis (GFC) emphasised the improved management of risk by financial institutions. As the Greeks, which depend on the BSM model, are used as tools to manage risk, and the GFC exposed the short comings of the BSM model, there is a need to improve the Greeks. This is achieved by improving the BSM model and considering the Greeks for the improved model.

\section{Piterbarg model}

The Piterbarg framework [10] implements three unique deterministic rates, namely the funding rate $\mathrm{rF}$ at which the derivative is funded, the collateral rate $\mathrm{rC}$ earned on the posted collateral, and the rate earned on a repurchase agreement $r R$ to price a derivative. In general we have that $r C \leq r R \leq r F$. The price, $V(t)$, at time $t$ of a derivative is then given by

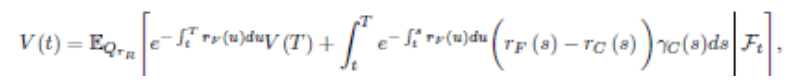

which, by applying the Feynman-Kac [3] theorem, can be rewrittenas

$$
\begin{aligned}
V(t) & =\mathbb{E}_{Q_{r_{n}}}\left[e^{-\int_{t}^{T} r_{G}(u) d u} V(T) \mid \mathcal{F}_{t}\right] \\
& -\mathbb{E}_{Q_{r_{n}}}\left[\int_{t}^{T} e^{-\int_{t}^{*} r_{C}(u) d u}\left(r_{F}(s)-r_{C}(s)\right)\left(V(s)-\gamma_{C}(s)\right) d s \mid \mathcal{F}_{t}\right],
\end{aligned}
$$

where $\gamma \mathrm{C}$ (s) equals the collateral amount paid at time $\mathrm{s} \in[\mathrm{t}, \mathrm{T}]$ and the expectation is taken under the measure at which the underlying asset grows at the rate $\mathrm{rR}$ earned on a repurchase agreement. This measure will be referred to as the QrR measure, and it is the measure under which no arbitrage exists when entering the replicating portfolio into a repurchase agreement. Additionally, if we assume that all the individual rates are equal to the constant risk-free interest rate, then the Piterbarg framework collapses back to the Black-Scholes- Merton framework.

The price of a derivative in the Piterbarg framework depends on the collateral amount paid at each point in time. If the trade is fully collateralised, so that at every time point $s \in[\mathrm{t}, \mathrm{T}]$ the collateral amount is equal to the value of the derivative,

$\gamma \mathrm{C}(\mathrm{s})=\mathrm{V}(\mathrm{s})$,

then from equation (3.2) the price of the derivative is given by

$$
V_{F C}(t)=\mathbb{E}_{Q_{r_{R}}}\left[e^{-\int_{t}^{T} r_{o}(u) d u} V(T) \mid \mathcal{F}_{t}\right] .
$$

If no collateral is paid throughout the lifetime of the derivative, such that

$$
\gamma_{C}(s)=0,
$$

for all $s \in[t, T]$, then from equation (3.1) the price of the derivative is given by

$$
V_{Z C}(t)=\mathbb{E}_{Q_{r_{n}}}\left[e^{-\int_{t}^{T} r y(u) d u} V(T) \mid \mathcal{F}_{t}\right] .
$$

Furthermore, if we assume that at every point in time $s \in[t, T]$ we pay a fractional amount $\theta$ of the uncollateralised trade as collateral, such that

$$
\mathbb{E}_{Q_{r n}}\left[\gamma_{C}(s)\right]=\mathbb{E}_{Q_{r n}}\left[\theta V_{Z C}(s)\right]=\mathbb{E}_{Q_{r n}}\left[\theta e^{-\int_{a}^{T} r_{p}(u) d u} V(T)\right],
$$

then, from equation (3.1), the price of the derivative is given by

$$
\begin{aligned}
& V_{P C(t)}=\mathrm{E}_{Q_{T_{n}}}\left[e^{-\int_{t}^{T} r_{r(u) d U_{U}}(T)} \mid \mathcal{F}_{t}\right]
\end{aligned}
$$

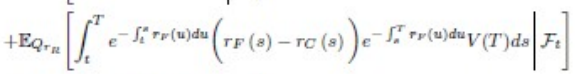

$$
\begin{aligned}
& =\mathbb{E}_{Q_{\tau_{R}}}\left[e^{-\int_{t}^{T} r_{\gamma}(u) d u_{V}(T)} \mid \mathcal{F}_{t}\right]
\end{aligned}
$$

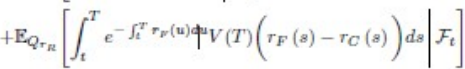

$$
\begin{aligned}
& =\mathrm{E}_{Q_{T_{n}}}\left[e^{-\int_{t}^{T} r_{F}(u) d u_{V}(T)}\left(1+\int_{t}^{T} \theta\left(r_{F}(s)-r_{C}(s)\right) d s \mid \mathcal{F}_{t}\right]\right.
\end{aligned}
$$

Form the above cases of collateral payments a closed form solution can be derived for the price of European options in the Piterbarg framework.

The purpose of this paper is to derive the closed form solution for the price of European option in the Piterbarg framework, and derive analytic solutions for the Greeks of the Piterbarg framework.

\section{The closed form solution for the price of European options in the Piterbarg framework}

The price of a zero collateral European call option in the Piterbarg framework is given by where F (ST) is the probability density function of the underlying asset $\mathrm{S}$ at time T. The integral is split into two parts, namely

$$
\begin{aligned}
& I_{1}=\int_{K}^{\infty} S_{T} d F\left(S_{T}\right), \\
& I_{2}=\int_{K}^{\infty} K d F\left(S_{T}\right) .
\end{aligned}
$$

Using the fact that the asset price process is log-normally distributed, it follows that

$$
\begin{aligned}
& I_{1}=\int_{K}^{\infty} S_{T} d F\left(S_{T}\right)=\mathbb{E}_{Q_{r n}}\left[S_{T} \mid S_{T}>K, \mathcal{F}_{t}\right]
\end{aligned}
$$

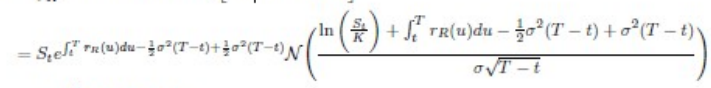

$$
\begin{aligned}
& =S_{t} e_{t}^{\int_{t}^{T} r_{n}\left(u_{1} d u\right.} \mathcal{N}\left(d_{1}\right),
\end{aligned}
$$

where $\mathrm{N}$ is the cummulative probability density function of a standard normal random variable, and

$$
d_{1}=\frac{\ln \left(\frac{S_{4}}{K}\right)+\int_{t}^{T} T_{R}(u) d u+\frac{1}{2} \sigma^{2}(T-t)}{\sigma \sqrt{T-t}} .
$$

Where

$$
d_{2}=d_{1}-\sigma \sqrt{T-t} .
$$

Therefore the price of a zero collateral European call option in the Piterbarg framework is given by

$$
V_{Z C}(t)=e^{-\int_{t}^{T} r_{F}(u) d u}\left(S_{t} \int_{t}^{\int_{t}^{T} r_{R}(u) d u} \mathcal{N}\left(d_{1}\right)-K \mathcal{N}\left(d_{2}\right)\right),
$$

where $\mathrm{d} 1$ and $\mathrm{d} 2$ are defined as above. The closed form solution of a zero collateral European put option is derived in a similar manner. The price of zero collateral European options in the Piterbarg framework can therefore be expressed as

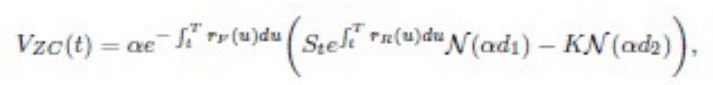

where $\alpha=1$ for a call option, and $\alpha=0$ for a put option. Analogously, the price of a fully collateralised European option in the Piterbarg framework is given by

$$
V_{F C}(t)=\alpha e^{-\int_{t}^{T} r_{\sigma}(u) d u}\left(S_{t} e^{\int_{t}^{T} r_{R}(u) d u} \mathcal{N}\left(\alpha d d_{1}\right)-K \mathcal{N}\left(\alpha d_{2}\right)\right) .
$$

Under the assumption of the partial collateral payments given in the previous section, and since Piterbarg's original work assumes that the interest rates are deterministic, the price of a partially collateralised European option is given by

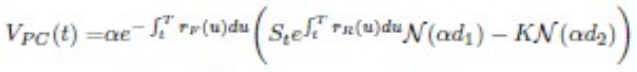

$$
\begin{aligned}
& \times\left(1+\int_{t}^{T} \theta\left(r_{F}(s)-r_{C}(s)\right) d s\right) .
\end{aligned}
$$

\section{The Greeks of the Piterbarg option pricing model}

The Greeks of the Piterbarg option pricing model are given below. The complete deriva- tions can be found in the appendix. First the Greeks are stated for zero collateral options, followed by the Greeks of the fully collateralised options, and lastly by the Greeks of the general case.

\subsection{Option Greeks:}

Delta:

$$
\begin{aligned}
& \cdot \frac{\partial V_{Z C, t}}{\partial S_{t}}=\alpha e^{-\int_{t}^{T}\left(r_{F}(u)-r_{R}(u)\right) d u} \mathcal{N}\left(\alpha d_{1}\right) . \\
& \text { • } \frac{\partial V_{F C, t}}{\partial S_{t}}=\alpha e^{-\int_{t}^{T}\left(r_{c}(u)-r_{R}(u)\right) d u} \mathcal{N}\left(\alpha d_{1}\right) . \\
& \text { • } \frac{\partial V_{P C, t}}{\partial S_{t}}=\alpha e^{-\int_{t}^{T}\left(r_{r}(\mathrm{u})-r_{n}(u)\right) d u} \mathcal{N}\left(\alpha d_{1}\right)\left(1+\int_{t}^{T} \theta\left(r_{F}(s)-r_{C}(s)\right) d s\right) .
\end{aligned}
$$

The delta of the Piterbarg option pricing framework gradually increases to either one or zero as the option reaches maturity, and measures the sensitivity of the option price with regards to the change of the underlying asset price. Unlike the Black-Scholes-Merton framework, the fully 
$\cdot \frac{\partial^{2} V_{Z C, t}}{\partial S_{t}^{2}}=\frac{1}{S_{t} \sigma \sqrt{T-t}} e^{-\int_{t}^{T}\left(r_{\mu}(u)-r_{n}(u)\right) d u} \mathcal{N}^{\prime}\left(\alpha d_{1}\right)$.

- $\frac{\partial^{2} V_{F C, t}}{\partial S_{t}^{2}}=\frac{1}{S_{t} \sigma \sqrt{T-t}} e^{-\int_{t}^{T}\left(r_{G}(u)-r_{R}(\mathrm{u})\right) d u} \mathcal{N}^{\prime}\left(\alpha d_{1}\right)$.

- $\frac{\partial^{2} V_{P C, t}}{\partial S_{t}^{2}}=\frac{1}{S_{t} \sigma \sqrt{T-t}} e^{-\int_{t}^{T}\left(r_{r}(u)-r_{n}(u)\right) d u} \mathcal{N}^{\prime}\left(\alpha d_{1}\right)\left(1+\int_{t}^{T} \theta\left(r_{F}(s)-r_{C}(s)\right) d s\right)$

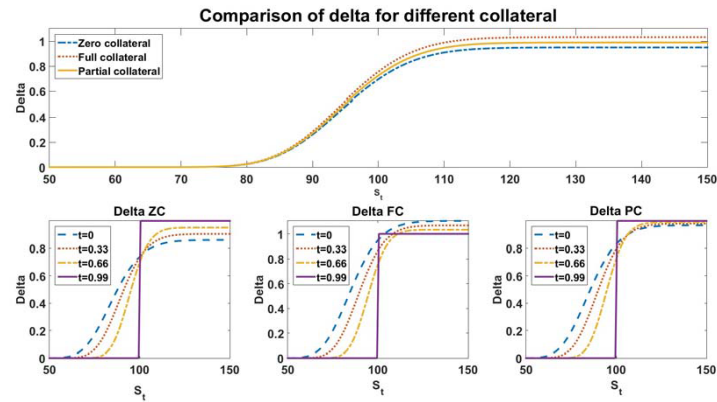

Figure 1: The delta of an European call option with strike $K=100$ plotted for different times versus the underlying asset price, for different cases of collateral payments. The option has a maturity of $\mathrm{T}=1$ year, and the volatility of the underlying is given by $\sigma=0.15$. The interest rates are assumed to be compounded continuously and constant and are given by $\mathrm{rC}=0.05, \mathrm{rR}=0.15$ and $\mathrm{rF}=0.3$.

The gamma measures the sensitivity of the delta with respect to changes in the under- lying assets price. As the option reaches maturity, the gamma of the option will become greatest at the 'at-the- money' position of the underlying asset. The gamma can be used to correct the hedging position for the convexity of the position.

Vega:

$$
\begin{aligned}
& \bullet \frac{\partial V_{Z C, t}}{\partial \sigma}=e^{-\int_{t}^{T} r_{y}(u) d u} K \mathcal{N}^{\prime}\left(\alpha d_{2}\right) \sqrt{T-t} . \\
& \bullet \frac{\partial V_{F C, t}}{\partial \sigma}=e^{-\int_{t}^{T} r_{C}(u) d u} K \mathcal{N}^{\prime}\left(\alpha d_{2}\right) \sqrt{T-t} . \\
& \bullet \frac{\partial V_{P C, t}}{\partial \sigma}=e^{-\int_{t}^{T} r_{r}(u) d u} K \mathcal{N}^{\prime}\left(\alpha d_{2}\right) \sqrt{T-t}\left(1+\int_{t}^{T} \theta\left(r_{F}(s)-r_{C}(s)\right) d s\right) .
\end{aligned}
$$

The option vega is always positive regardless of whether the option is a put or a call.

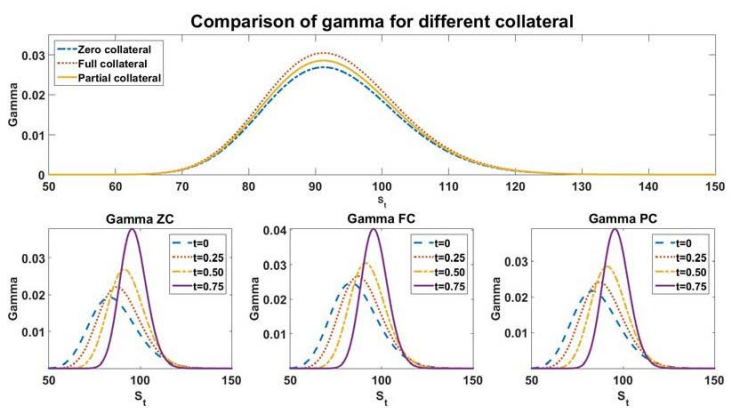

Figure 2: The gamma of an European call option with strike $\mathrm{K}=100$ plotted for different times versus the underlying asset price, for different cases of collateral payments. The option has a maturity of $\mathrm{T}=1$ year, and the volatility of the underlying is given by $\sigma=0.15$. The interest rates are assumed to be compounded continuously and constant and are given by $\mathrm{rC}$ $=0.05, \mathrm{rR}=0.15$ and $\mathrm{rF}=0.3$.

vega measures the sensitivity of the option price with respect to the underlying assets volatility. As the time to maturity decreases, the option vega will become closer to zero, as the volatility of the underlying asset will become less important over a shorter period of time, since the underlying will be less likely to move drastically in a shorter timespan compared to a longer time span.

Theta:

$$
\begin{aligned}
\cdot \frac{\partial V_{Z C, t}}{\partial t} & =\alpha e^{-\int_{t}^{T}\left(r_{\nu}(u)-r_{R}(\mathrm{u})\right) d u}\left(r_{F}(t)-r_{R}(t)\right) S_{t} \mathcal{N}\left(\alpha d_{1}\right) \\
& -e^{-\int_{t}^{T} r_{\nu}(u) d u}\left(\alpha K \mathcal{N}\left(\alpha d_{2}\right) r_{F}(t)-K \mathcal{N}^{\prime}\left(\alpha d_{2}\right) \frac{\sigma}{2 \sqrt{T-t}}\right) . \\
\bullet \frac{\partial V_{F C, t}}{\partial t} & =\alpha e^{-\int_{t}^{T}\left(r_{C}(\mathrm{u})-r_{n}(u)\right) d u}\left(r_{C}(t)-r_{R}(t)\right) S_{t} \mathcal{N}\left(\alpha d_{1}\right) \\
& -e^{-\int_{t}^{T} r_{C}(u) d u}\left(\alpha K \mathcal{N}\left(\alpha d_{2}\right) r_{C}(t)-K \mathcal{N}^{\prime}\left(\alpha d_{2}\right) \frac{\sigma}{2 \sqrt{T-t}}\right) .
\end{aligned}
$$

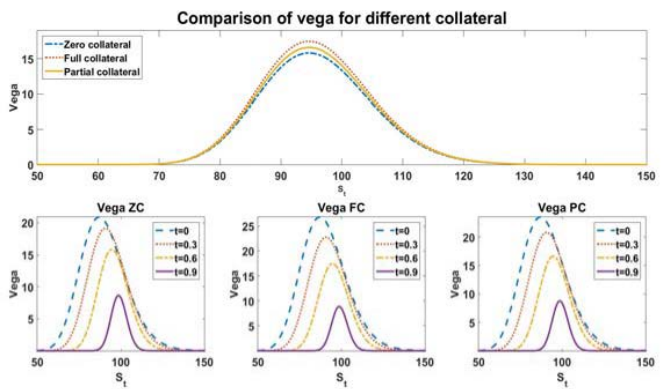

Figure 3: The vega of an European call option with strike $\mathrm{K}=100$ plotted for different times versus the underlying asset price, for different cases of collateral payments. The option has a maturity of $\mathrm{T}=1$ year, and the volatility of the underlying is given by $\sigma=0.15$. The interest rates are assumed to be compounded continuously and constant and are given by $\mathrm{rC}$ $=0.05, \mathrm{rR}=0.15$ and $\mathrm{rF}=0.3$.

$$
\begin{aligned}
\cdot \frac{\partial V_{P C, t}}{\partial t} & =\left[\alpha e^{-\int_{t}^{T}\left(r_{v}(u)-r_{n}(u)\right) d u}\left(r_{F}(t)-r_{R}(t)\right) S_{t} \mathcal{N}\left(\alpha d_{1}\right)\right. \\
& \left.-e^{-\int_{t}^{T} r_{v}(u) d u}\left(\alpha K \mathcal{N}\left(\alpha d_{2}\right) r_{F}(t)-K \mathcal{N}^{\prime}\left(\alpha d_{2}\right) \frac{\sigma}{2 \sqrt{T-t}}\right)\right] \\
& \times\left(1+\int_{t}^{T} \theta\left(r_{F}(s)-r_{C}(s)\right) d s\right) \\
& +e^{-\int_{t}^{T} r_{v}(u) d u}\left(S_{t} e^{\int_{t}^{T} r_{n}(u) d u} \mathcal{N}\left(\alpha d_{1}\right)-K \mathcal{N}\left(\alpha d_{2}\right)\right) \theta\left(r_{C}(t)-r_{F}(t)\right) .
\end{aligned}
$$

Theta measures the time decay of the option. As the underlying asset price becomes smaller than the strike price, the value of theta approaches zero since the call option is less likely to gain any value throughout time, as the likelihood of the asset price crossing the strike price becomes less. If the position on the option is long, then theta is short. In other words the portfolio will lose value over time, if everything remains constant. Rho rF :

$$
\text { - } \frac{\partial V_{Z C, t}}{\partial r_{F}}=-\alpha(T-t) e^{-\int_{t}^{T} r_{\nu}(u) d u}\left(S_{t} e^{\int_{t}^{T} r_{u}(u) d u} \mathcal{N}\left(\alpha d_{1}\right)-K \mathcal{N}\left(\alpha d_{2}\right)\right) .
$$

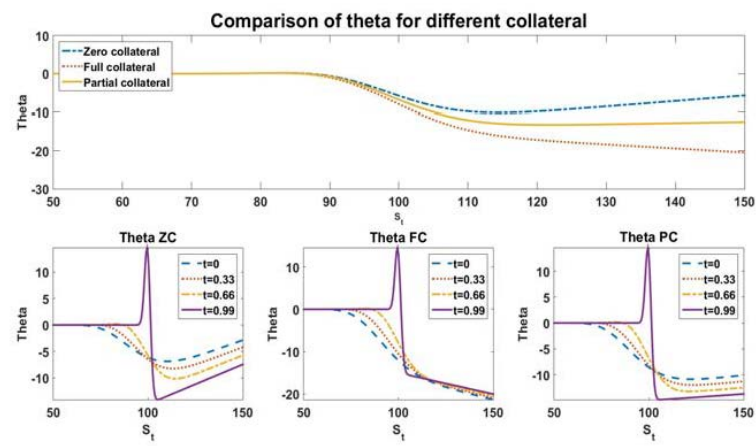

Figure 4: The theta of an European call option with strike $K=100$ plotted for different times versus the underlying asset price, for different cases of collateral payments. The option has a maturity of $\mathrm{T}=1$ year, and the volatility of the underlying is given by $\sigma=0.15$. The interest rates are assumed to be compounded continuously and constant and are given by $\mathrm{rC}=$ $0.05, \mathrm{rR}=0.15$ and $\mathrm{rF}=0.3$.

$$
\begin{aligned}
\cdot \frac{\partial V_{F C, t}}{\partial T_{F}} & =0 . \\
\cdot \frac{\partial V_{P C, t}}{\partial r_{F}} & =-\alpha(T-t) e^{-\int_{t}^{T} r_{F}(u) d u}\left(S_{t} e^{\int_{t}^{T} r_{n}(u) d u} \mathcal{N}\left(\alpha d_{1}\right)-K \mathcal{N}\left(\alpha d_{2}\right)\right) \\
& \times\left(1+\int_{t}^{T} \theta\left(r_{F}(s)-r_{C}(s)\right) d s\right) \\
& +e^{-\int_{t}^{T} r_{r}(u) d u}\left(S_{t} e_{t}^{\int_{t}^{T} r_{n}(u) d u} \mathcal{N}\left(\alpha d_{1}\right)-K \mathcal{N}\left(\alpha d_{2}\right)\right) \theta(T-t) .
\end{aligned}
$$

The rho with respect to the funding rate $\mathrm{rF}$ of an option in which full collateral is paid continuously is zero since the option price is not a function of the funding rate. Therefore only the zero collateral and partial collateral option prices are regarded. The funding rate is used to discount the future expected value of the derivative, and therefore becomes zero at the option maturity. Before maturity, an increase in the funding rate will cause the price of the option to drop. Therefore the rho with respect to the funding rate is negative. 


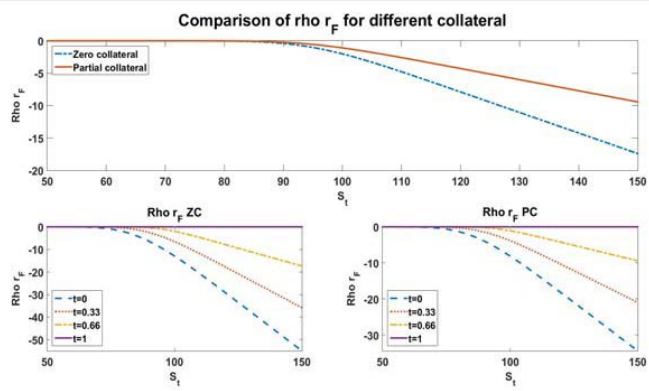

Figure 5: The rho with respect to the funding rate $\mathrm{rF}$ of an European call option with strike $\mathrm{K}=100$ plotted for different times versus the underlying asset price, for different cases of collateral payments. The option has a maturity of $\mathrm{T}=1$ year, and the volatility of the underlying is given by $\sigma=$ 0.15 . The interest rates are assumed to be compounded continuously and constant and are given by $\mathrm{rC}=0.05, \mathrm{rR}=0.15$ and $\mathrm{rF}=0.3$.

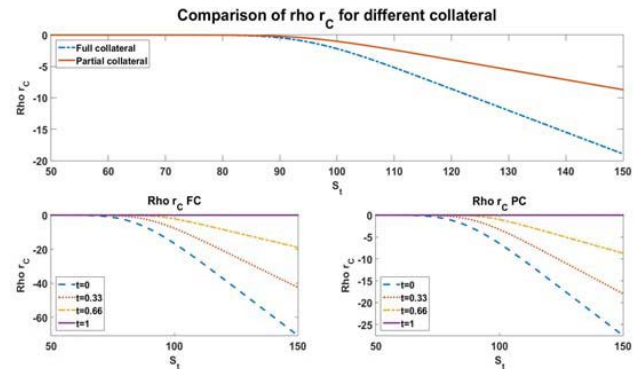

Figure 6: The rho with respect to the collateral rate $\mathrm{rC}$ of an European call option with strike $\mathrm{K}=100$ plotted for different times versus the underlying asset price, for different cases of collateral payments. The option has a maturity of $\mathrm{T}=1$ year, and the volatility of the underlying is given by $\sigma=$ 0.15 . The interest rates are assumed to be compounded continuously and constant and are given by $\mathrm{rC}=0.05, \mathrm{rR}=0.15$ and $\mathrm{rF}=0.3$.

Similarly to the rho with respect to the funding rate, the rho with respect to the collateral race $\mathrm{rC}$ is zero for the zero collateral option price, since the option price is not a function the collateral rate. The future expected payoff of the derivative is discounted at the collateral rate in the fully collateralised option price, therefore an increase in the collateral rate reduces the price of the option, and rho with respect to the collateral rate is negative.

Rho rR:

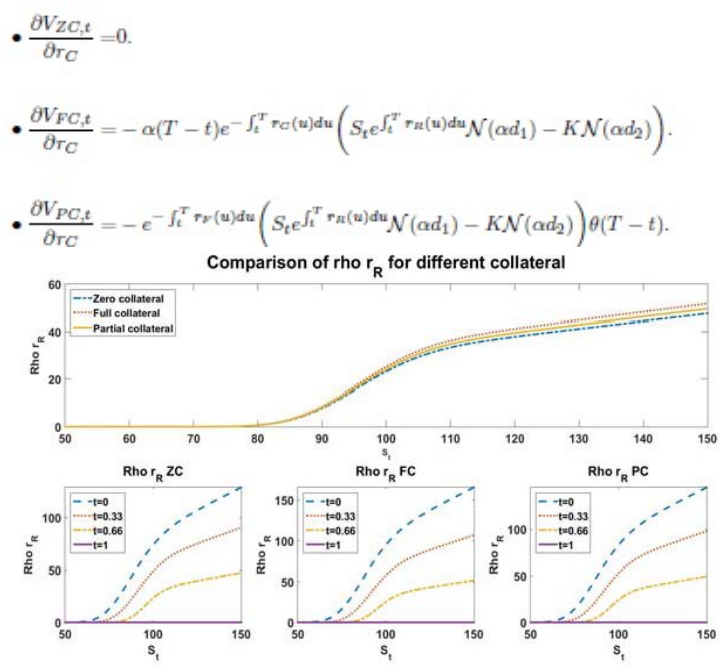

Figure 7: The rho with respect to the repurchase rate rR of an European call option with strike $\mathrm{K}=100$ plotted for different times versus the underlying asset price, for different cases of collateral payments. The option has a maturity of $\mathrm{T}=1$ year, and the volatility of the underlying is given by $\sigma=$ 0.15 . The interest rates are assumed to be compounded continuously and constant and are given by $\mathrm{rC}=0.05, \mathrm{rR}=0.15$ and $\mathrm{rF}=0.3$.

In Piterbarg's framework the repurchase plays an important role, since the measure in which the derivative is priced is in the QrR measure at which the underlying asset drifts at the repurchase rate, which means that the returns of the underlying asset in the QrR measure are equal to the repurchase rate. This means that an increase in the repurchase rate will increase the future expected value of the asset, which in turn increases the expected call option payoff. Therefore rho with respect to the repurchase rate is positive for call options.

\section{Derivation of the Greeks}

The Greeks of the fully collateralised options and the zero collateral options are derived in a identical fashion, by replacing the funding rate $\mathrm{rF}$ of the zero collateral Greeks by the collateral rate $\mathrm{rC}$ of the fully collateralised Greeks. Therefore only the zero collateral option Greeks are derived, and the fully collateralised option Greeks can be foudn by replacing $\mathrm{rF}$ by $\mathrm{rC}$. First, a preliminary result which is needed in the derivation of the Greeks is introduced in the following lemma. Lemma 6.1. Given that

$d_{2}=d_{1}-\sigma \sqrt{T-t}$, where

$$
d_{1}=\frac{\ln \left(\frac{S_{1}}{K}\right)+\int_{t}^{T} T R(u) d u+\frac{1}{2} \sigma^{2}(T-t)}{\sigma \sqrt{T-t}},
$$

the following result holds:

$$
S_{t} \mathcal{N}^{\prime}\left(\alpha d_{1}\right) e^{\int_{t}^{T} r_{R}(u) d u}=K \mathcal{N}^{\prime}\left(\alpha d_{2}\right) .
$$

Proof.

$$
\begin{aligned}
\mathcal{N}^{\prime}\left(\alpha d_{1}\right) & =\mathcal{N}^{\prime}\left(\alpha d_{2}+\alpha \sigma \sqrt{T-t}\right)=\frac{1}{\sqrt{2 \pi}} e^{-\frac{1}{2}\left(\alpha d_{2}+\alpha \sigma \sqrt{T-t}\right)^{2}} \\
& =\frac{1}{\sqrt{2 \pi}} e^{-\frac{1}{2} \alpha^{2}\left(d_{2}^{2}+2 d_{2} \sigma \sqrt{T-t}+\sigma^{2}(T-t)\right.} \\
& =\mathcal{N}^{\prime}\left(\alpha d_{2}\right) e^{-\frac{\ln \left(\frac{S^{\prime}}{\mathrm{t}}\right)+\int_{t}^{T} r_{R}(u) d s-\frac{1}{2} \sigma^{2}(T-t)}{\sigma \sqrt{T-t}} \sigma \sqrt{T-t}-\frac{1}{2} \sigma^{2}(T-t)} \\
& =\mathcal{N}^{\prime}\left(\alpha d_{2}\right) \frac{K}{S_{t}} e^{-\int_{t}^{T} r_{R}(\mathrm{u}) d u},
\end{aligned}
$$

which gives us the identity of

$$
\begin{aligned}
& S_{t} \mathcal{N}^{\prime}\left(\alpha d_{1}\right) \int^{T_{t}^{T} r_{R}(u) d u}=K \mathcal{N}^{\prime}\left(\alpha d_{2}\right) . \\
& \text { Delta: } \\
& \frac{\partial V_{Z C, t}}{\partial S_{t}}=\alpha e^{-\int_{t}^{T} r_{v}(\mathrm{u}) d u}\left(e^{\int_{t}^{T} r_{r}(u) d u} \mathcal{N}\left(\alpha d_{1}\right)+S_{t} e^{-\int_{t}^{T} r_{r}(\mathrm{u}) d u} \mathcal{N}^{\prime}\left(\alpha d_{1}\right) \frac{\partial\left(\alpha d_{1}\right)}{\partial S_{t}}\right. \\
& \left.-K \mathcal{N}^{\prime}\left(\alpha d_{2}\right) \frac{\partial\left(\alpha d_{2}\right)}{\partial S_{t}}\right) \\
& \text { The result from Lemma } 6.1 \text { is used to rewrite the above equation as } \\
& \frac{\partial V_{Z C, t}}{\partial S_{t}}=\alpha e^{-\int_{t}^{T} r_{r}(\mathbf{u}) d u}\left(e^{\int_{t}^{T} r_{R}(u) d u} \mathcal{N}\left(\alpha d_{1}\right)+K \mathcal{N}\left(\alpha d_{2}\right) \frac{\partial\left(\alpha d_{2}\right)}{\partial S_{t}}\right. \\
& \left.-K \mathcal{N}^{\prime}\left(\alpha d_{2}\right) \frac{\partial\left(\alpha d_{2}\right)}{\partial S_{t}}\right),
\end{aligned}
$$

which gives us the desired result.

$$
\begin{aligned}
& \text { Gamma: } \\
& \begin{aligned}
\frac{\partial^{2} V_{Z C, t}}{\partial S_{t}^{2}} & =\alpha e^{-\int_{t}^{T}\left(r_{\nu}(\mathrm{u})-r_{n}(u)\right) d u} \mathcal{N}^{\prime}\left(\alpha d_{1}\right) \frac{\partial\left(\alpha d_{2}\right)}{\partial S_{t}} \\
& =\alpha e^{-\int_{t}^{T}\left(r_{p}(\mathrm{u})-r_{n}(u)\right) d u} \mathcal{N}^{\prime}\left(\alpha d_{1}\right) \frac{\alpha}{S_{t} \sigma \sqrt{T-t}} \\
& =e^{-\int_{t}^{T}\left(r v(u)-r_{R}(\mathrm{u})\right) d u^{\prime} \mathcal{N}^{\prime}\left(\alpha d_{1}\right)} \frac{1}{S_{t} \sigma \sqrt{T-t}},
\end{aligned}
\end{aligned}
$$

where we used the fact that $\alpha^{2}=1$, irrelevant of whether the option is a put or a call.

Vega:

$$
\frac{\partial V_{Z C, t}}{\partial \sigma}=\alpha e^{-\int_{t}^{T} r_{p}(u) d u}\left(S_{t} e_{t}^{\int_{t}^{T} r_{n}(u) d u_{\mathcal{N}} \mathcal{N}^{\prime}\left(\alpha d_{1}\right)} \frac{\partial\left(\alpha d_{1}\right)}{\partial \sigma}-K \mathcal{N}^{\prime}\left(\alpha d_{2}\right) \frac{\partial\left(\alpha d_{2}\right)}{\partial \sigma}\right)
$$$$
\text { Since } \alpha d_{2}=\alpha d_{1}-\alpha \sigma \sqrt{T-t} \text {, we have that }
$$$$
\frac{\partial\left(\alpha d_{2}\right)}{\partial \sigma}=\frac{\partial\left(\alpha d_{1}\right)}{\partial \sigma}-\alpha \sqrt{T-t}
$$

Further more, the partial derivative of $\left(\alpha d_{1}\right)$ with respect to $\sigma$ is given by

$$
\begin{aligned}
\frac{\partial\left(\alpha d_{1}\right)}{\partial \sigma} & =\alpha \frac{\partial}{\partial \sigma}\left(\frac{\ln \left(\frac{S_{A}}{K}\right)+\int_{t}^{T} r_{R}(u) d u+\frac{1}{2} \sigma^{2}(T-t)}{\sigma \sqrt{T-t}}\right) \\
& =\alpha\left(\sqrt{T-t}-\frac{\ln \left(\frac{S_{T}}{K}\right)+\int_{t}^{T} r_{R}(u) d u+\frac{1}{2} \sigma^{2}(T-t)}{\sigma^{2} \sqrt{T-t}}\right) .
\end{aligned}
$$

Therefore

$$
\begin{aligned}
& \frac{\partial V_{Z C, t}}{\partial \sigma}=\alpha e^{-\int_{t}^{T} r_{r}(u) d u}\left[\alpha S_{t} e_{t}^{T} r_{R}(u) d u \mathcal{N}^{\prime}\left(\alpha d_{1}\right)\left(\frac{\frac{1}{2} \sigma^{2}(T-t)-\ln \left(\frac{S_{L}}{K}\right)-\int_{t}^{T} r_{R}(u) d u}{\sigma^{2} \sqrt{T-t}}\right)\right. \\
& \left.-\alpha K \mathcal{N}^{\prime}\left(\alpha d_{2}\right)\left(-\frac{\ln \left(\frac{S_{S}}{K}\right)+\int_{t}^{T} r_{R}(u) d u+\frac{1}{2} \sigma^{2}(T-t)}{\sigma^{2} \sqrt{T-t}}\right)\right] .
\end{aligned}
$$

Using the result from Lemma 6.1 and the fact that $\alpha^{2}=1$, we obtain 
Theta:

$$
\begin{aligned}
& \frac{\partial V_{Z C, t}}{\partial t}=\alpha \frac{\partial}{\partial t}\left[e^{-\int_{t}^{T}\left(r_{v}(u)-r_{R}(u)\right) d d_{S}} S_{t} \mathcal{N}\left(\alpha d_{1}\right)-K e^{-\int_{t}^{T} r_{r}(\mathrm{u}) d u} \mathcal{N}\left(\alpha d_{2}\right)\right] \\
& =\alpha \frac{\partial}{\partial t}\left(e^{-\int_{t}^{T}\left(r_{\nu}(u)-r_{n}(u)\right) d u}\right) S_{t} \mathcal{N}\left(\alpha d_{1}\right)+\alpha e^{-\iint_{t}^{T}\left(r y(u)-r_{R}(u)\right) d u} S_{t} \mathcal{N}^{\prime}\left(\alpha d_{1}\right) \frac{\partial\left(\alpha d_{1}\right)}{\partial t} \\
& -\alpha K \mathcal{N}\left(\alpha d_{2}\right) \frac{\partial}{\partial t}\left(e^{-\int_{t}^{T} r_{r}(u) d u}\right)-\alpha K e^{-\int_{t}^{T} r_{\nu}(u) d u} \mathcal{N}^{\prime}\left(\alpha d_{2}\right) \frac{\partial\left(\alpha d_{2}\right)}{\partial t} \\
& =\alpha \frac{\partial}{\partial t}\left(-\int_{t}^{T}\left(r_{F}(u)-r_{R}(u)\right) d u\right) e^{-\int_{t}^{T}\left(r_{r_{F}}(u)-r_{R}(u)\right) d u} S_{t} \mathcal{N}\left(\alpha d_{1}\right)
\end{aligned}
$$

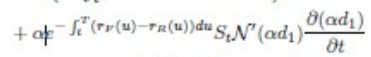

$$
\begin{aligned}
& -\alpha K \mathcal{N}\left(\alpha d_{2}\right) \frac{\partial}{\partial t}\left(-\int_{t}^{T} r_{F}(u) d u\right) e^{-\int_{t}^{T} r_{r}(u) d u}
\end{aligned}
$$

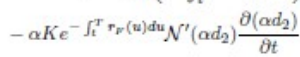

Under the assumption that the interest rate function given by $\mathrm{rF}$ (and similarly for $\mathrm{rC}$ and $\mathrm{rR}$ ) is continuous and the partial derivative of the function with respect to time exists, the following identity holds:

$$
\begin{aligned}
& \qquad \frac{\partial}{\partial t}\left(\int_{t}^{T} r_{F}(u) d u\right) \\
& \text { Proof. } \\
& \begin{aligned}
\frac{\partial}{\partial t}\left(\int_{t}^{T} r_{F}(u) d u\right) & =\lim _{\Delta t \rightarrow 0} \frac{1}{\Delta t}\left[\int_{t+\Delta t}^{T} r_{F}(u) d u-\int_{t}^{T} r_{F}(u) d u\right] \\
& =\lim _{\Delta t \rightarrow 0} \frac{1}{\Delta t} \int_{t+\Delta t}^{t} r_{F}(u) d u \\
& =\lim _{\Delta t \rightarrow 0} \frac{1}{\Delta t}\left[-r_{F}(t) \Delta t+\mathcal{O}\left(\Delta t^{2}\right)\right] \\
& =-r_{F}(t) .
\end{aligned}
\end{aligned}
$$

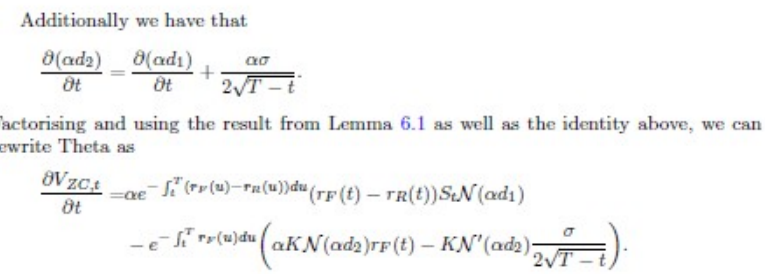

Rho $r_{F}$ :

$$
\begin{aligned}
& \frac{\partial V_{Z C_{C}, t}}{\partial \tau_{F}}=\alpha \frac{\partial}{\partial r_{F}}\left(e^{-\int_{t}^{T} r_{y}(u) d u}\right)\left(S_{t} e^{f_{t}^{T} r_{n}(u) d u} \mathcal{N}\left(\alpha d_{1}\right)-K \mathcal{N}\left(\alpha d_{2}\right)\right)
\end{aligned}
$$

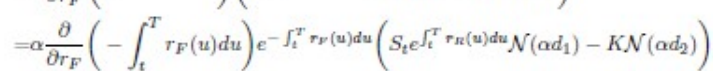

Under the assumption that the interest rate function given by $r_{F}$ is continuous, we cal write

$$
\begin{aligned}
& \frac{\partial V_{Z C, t}}{\partial \tau_{F}}=\alpha\left(-\int_{t}^{T} \frac{\partial}{\partial r_{F}} r_{F}(u) d u\right) e^{-\int_{t}^{T} r_{y}(u) d u}\left(S_{t} e^{\int_{t}^{T} r_{R}(u) d u} \mathcal{N}\left(\alpha d_{1}\right)-K \mathcal{N}\left(\alpha d_{2}\right)\right) \\
& =-\alpha(T-t) e^{-\int_{t}^{T} r_{r}(\mathrm{u}) d u}\left(S_{t} e^{\iint_{t}^{T} r_{R}(u) d u} \mathcal{N}\left(\alpha d_{1}\right)-K \mathcal{N}\left(\alpha d_{2}\right)\right) .
\end{aligned}
$$

Rho rC :

Similarly to how the fully collateralised option price is not a function of the funding inter- est rate, the zero collateral option price is not a function of the collateral rate. Therefore the partial derivative of the zero collateral option price with respect to the collateral rate $\mathrm{rC}$ is zero. Likewise, the partial derivative of the fully collateralised option price with respect to the funding rate is equal to zero, where as the partial derivative of the fully collateralised option with respect to the collateral rate $\mathrm{rC}$ is equal to the partial deriva- tive of the zero collateral option price with respect to the funding rate, but replacing the funding rate $\mathrm{rF}$ by the collateral rate $\mathrm{rC}$. Rho rR:

$$
\begin{aligned}
\frac{\partial V_{Z C, t}}{\partial r_{R}}=\alpha e^{-\int_{t}^{T} r_{r}(\mathrm{u}) d u}[ & S_{t} \frac{\partial}{\partial r_{R}}\left(\int_{t}^{T} r_{R}(u) d u\right) e^{\int_{t}^{T} r_{R}(\mathrm{u}) d u} \mathcal{N}\left(\alpha d_{1}\right) \\
& \left.+S_{t} e_{t}^{\int_{t}^{T} r_{u}(u) d u} \mathcal{N}^{\prime}\left(\alpha d_{1}\right) \frac{\partial\left(\alpha d_{1}\right)}{\partial r_{R}}-K \mathcal{N}^{\prime}\left(\alpha d_{2}\right) \frac{\partial\left(\alpha d_{2}\right)}{\partial d_{2}}\right] .
\end{aligned}
$$

By definition of $d_{1}$ and $d_{2}$, we have that

$$
\frac{\partial \alpha d_{2}}{\partial r_{R}}=\frac{\partial \alpha d_{1}}{\partial r_{R}}=\alpha \frac{\partial}{\partial r_{R}}\left(\frac{\int_{t}^{T} r R(u) d u}{\sigma \sqrt{T-t}}\right)=\alpha \frac{\int_{t}^{T} \frac{\partial}{\partial r_{R}} r R(u) d u}{\sigma \sqrt{T-t}}=\alpha \frac{\sqrt{T-t}}{\sigma},
$$

where we used the usual continuity assumption of the interest rate function. Therefore

$$
\begin{aligned}
\frac{\partial V_{Z C, t}}{\partial r_{R}}=\alpha e^{-\int_{t}^{T} r_{r(u) d u}} & \left(S_{t}(T-t) e^{\int_{t}^{T} r_{R}(u) d u} \mathcal{N}\left(\alpha d_{1}\right)-K \mathcal{N}^{\prime}\left(\alpha d_{2}\right) \frac{\alpha \sqrt{T-t}}{\sigma}\right. \\
& \left.+S_{t} e^{\int_{t}^{T} r_{R}(u) d u} \mathcal{N}^{\prime}\left(\alpha d_{1}\right) \frac{\alpha \sqrt{T-t}}{\sigma}\right) .
\end{aligned}
$$

Using the identity from Lemma 6.1 the above equation can be rewritten as

$$
\frac{\partial V_{Z C, t}}{\partial \tau_{R}}=\alpha e^{-\int_{t}^{T} r_{r}(\mathrm{u}) d u} S_{t}(T-t) e^{f_{t}^{T} r u(u) d u} \mathcal{N}\left(\alpha d_{1}\right) .
$$

\section{2ivations for the partially collateralised options}

The Greeks of the partially collateralised options are derived in a similar fashion to the Greeks of the zero collateral option prices. The delta, gamma, vega and rho with respect to the repurchase rate $r R$ of the partially collateralised option are identical to the zero collateral case with the addition of multiplying the term

$$
\left(1+\int_{t}^{T} \theta\left(r_{F}(s)-r_{C}(s)\right) d s\right),
$$

since the additional term is not a function of the partial derivatives of those Greeks. Theta, rho with respect to the funding rate $\mathrm{rF}$ and with respect to the collateral rate $\mathrm{rC}$ will be derived since the additional term given above is a function of thesevariables.

Theta:

To derive theta for the partially collateralised option the chain rule is implemented by noting that the option price is equal to the zero collateral option price multiplied the term

$$
\left(1+\int_{t}^{T} \theta\left(r_{F}(s)-r_{C}(s)\right) d s\right)
$$

The partial derivative of the additional term with respect to time is given by

$$
\frac{\partial}{\partial t}\left(1+\int_{t}^{T} \theta\left(r_{F}(s)-r_{C}(s)\right) d s\right)=\frac{\partial}{\partial t} \int_{t}^{T} \theta\left(r_{F}(s)-r_{C}(s)\right) d s .
$$

The continuity assumption of the interest rates and the identity used to derive theta for the zero and fully collateralised options gives us the following result:

$$
\begin{aligned}
\frac{\partial}{\partial t} \int_{t}^{T} \theta\left(r_{F}(s)-r C(s)\right) d s & =\int_{t}^{T} \theta \frac{\partial}{\partial t}\left(r_{F}(s)-r_{C}(s)\right) d s \\
& =\theta\left(r_{C}(t)-r_{F}(t)\right) .
\end{aligned}
$$

In conjunction with the chain rule, we obtain the desired result Rho rF :

The derivation uses similar arguments as the derivation of theta for the partially collat- eralised options. We use the chain rule and the fact that

$$
\begin{aligned}
\frac{\partial}{\partial T_{F}}\left(1+\int_{t}^{T} \theta\left(r_{F}(s)-r_{C}(s)\right) d s\right) & =\frac{\partial}{\partial T_{F}} \int_{t}^{T} \theta\left(r_{F}(s)-r_{C}(s)\right) d s \\
& =\int^{T} \theta d s=\theta(T-t),
\end{aligned}
$$

to obtain the desired result.

Rho rC :

The derivation uses similar arguments as the derivation of rho with respect to the funding rate $\mathrm{rF}$ for the partially collateralised options. We use the chain rule and the fact that

$$
\begin{aligned}
\frac{\partial}{\partial T_{C}}\left(1+\int_{t}^{T} \theta\left(r_{F}(s)-r_{C}(s)\right) d s\right) & =\frac{\partial}{\partial T_{C}} \int_{t}^{T} \theta\left(r_{F}(s)-r_{C}(s)\right) d s \\
& =-\int_{t}^{T} \theta d s=-\theta(T-t),
\end{aligned}
$$

to obtain the desired result.

\section{References}

[1] F. Black, M. Scholes, The Pricing of Options and Corporate Liabilities, J. of Polit. Econ. 81 (1973) 637-654

[2] Clewlow, L., Hodges, S.: Optimal delta-hedging under transactions costs. Journal of Economic Dynamics and Control 21, 1353-1376 (1997).

[3] P. Del Moral, Feynman-Kac Formulae, Springer, New York, 2004.

[4] Hull, J. Options, Futures and Other Derivatives (7th ed.)", New Jersey, Prentice Hall, 2009.

[5] Hull, J. Fundamentals of Corporate Finance (9th ed.)”. McGraw Hill, 2010.

[6] Hull, J., Options, futures, and other derivatives, Pearson Education Limited, Har- low, (2012a), Eighth edition.

[7] CB Hunzinger, CCA Labuschagne An Overview of the Black-Scholes-Merton Model After the 2008 Credit Crisis - In: Econometrics of Risk, 41-52, Springer 2015

[8] CCA Labuschagne, ST von Boetticher, Dupire's formulas in the Piterbarg option pricing model North American Journal of Economics and Finance 38 (2016) 148- 162.

[9] Merton, R.: Theory of Rational Option Pricing. Bell Journal of Economics and Management Science 4, 141-183 (1973).

[10] V. Piterbarg, Funding beyond discounting: Collateral agreements and derivatives pricing, Risk Mag. 23 (2010) 97-102. 